\title{
Quaderni
}

QUADERNI Communication, technologies, pouvoir

85 | Automne 2014

La régulation de la qualité dans le secteur de la santé

\section{La construction de la valeur de marque des universités à travers les réseaux sociaux. Le cas de Facebook}

\section{Pablo Medina Aguerrebere}

\section{OpenEdition}

\section{Journals}

Édition électronique

URL : http://journals.openedition.org/quaderni/841

DOI : 10.4000/quaderni.841

ISSN : 2105-2956

Éditeur

Les éditions de la Maison des sciences de l'Homme

\section{Édition imprimée}

Date de publication : 5 octobre 2014

Pagination : 85-100

Référence électronique

Pablo Medina Aguerrebere, "La construction de la valeur de marque des universités à travers les réseaux sociaux. Le cas de Facebook », Quaderni [En ligne], 85 | Automne 2014, mis en ligne le 05 octobre 2016, consulté le 01 mai 2019. URL : http://journals.openedition.org/quaderni/841 ; DOI : 10.4000/quaderni.841

Ce document a été généré automatiquement le 1 mai 2019.

Tous droits réservés 


\title{
La construction de la valeur de marque des universités à travers les réseaux sociaux. Le cas de Facebook
}

\author{
Pablo Medina Aguerrebere
}

1 Les réseaux sociaux constituent une opportunité intéressante pour les universités qui souhaitent diffuser leur image de marque au niveau international, ce qui peut influencer positivement sur leur réputation organisationnelle et académique. La concurrence internationale existante dans le milieu universitaire, la baisse de la natalité dans certains pays, le déficit public touchant les universités appartenant à l'État, le développement d'une attitude proactive chez les étudiants en ce qui concerne la consommation des médias, ainsi que la promotion de la culture de la communication institutionnelle au sein des universités sont autant de facteurs qui poussent ces organisations à parier sur la gestion professionnelle de la communication online 2.0, autrement dit, l'usage des ré seaux sociaux (Facebook, Youtube, etc.) comme un outil de communication institutionnelle.

2 Dans ce contexte, on peut se poser cette question : quel est l'impact de la communication 2.0 sur la construction de marque des universités?

3 L'objectif de cet article est d'analyser le vrai impact des réseaux sociaux sur la construction de la valeur de marque des universités. Autrement dit, l'article s'oriente à savoir si les applications telles que Facebook, Twitter ou Youtube représentent une opportunité réelle pour diffuser et ainsi construire la valeur de marque des universités, ou s'il s'agit plutôt d'un media secondaire. L'article développe plusieurs questions de recherche, notamment le rôle de la valeur de marque, l'identité, l'image de marque et la réputation, ainsi que l'influence des réseaux sociaux, notamment Facebook, sur la construction et divulgation de ces éléments identitaires dans le milieu des universités. Afin de mieux comprendre cette réalité, l'article analyse, en premier lieu, le développe ment des marques universitaires à travers les réseaux sociaux : et en deuxième lieu, avant les conclusions, on présente une étude quantitative sur le page Facebook des 100 meilleures universités au niveau international. 


\section{La construction de marques universitaires à travers les réseaux sociaux}

\section{Définition de la valeur de marque}

4 L'intérêt des organisations pour la rentabilité financière mène le citoyen à devenir très sceptique envers elles (Ählström, 2010). Néanmoins, les résultats obtenus par chaque institution déterminent les évaluations faites par les consommateurs, autrement dit, ces évaluations dépendent de la puissance des associations prédictives établies entre les résultats organisationnels et le nom de marque de l'institution (Janiszewski et Van Osselaer, 2000). Ainsi, le fonctionnement de l'organisation influence sur les perceptions des citoyens, d'où l'importance que l'organisation instaure des mécanismes lui permettant de transmettre à travers ses comportements sa vraie identité institutionnelle. Et cette identité est toujours en rapport avec le comportement organisationnel ainsi qu'avec la communication (Balmer, 2008), d'où l'intérêt que les organisations gèrent la communication institutionnelle d'une manière stratégique et professionnelle.

5 Les universités, à l'instar des autres organisations, parient sur la gestion de la communication institutionnelle comme stratégie leur permettant de gérer et diffuser une identité de marque cohérente avec les expectatives de leurs stakeholders (étudiants, professeurs, autorités publiques, médias, etc.). Le concept d'identité de marque fait référence à la vision, la culture, le positionnement, la personnalité, la capacité relationnelle et les images actuelles et souhaitées qu'ont les parties prenantes de l'institution (Harris et De Chernatony, 2001). D'ailleurs, l'identité de marque d'une organisation, comme par exemple une université, est déterminée, d'une part, par les valeurs et les comportements de ses employés (Wilson, 2001), et d'une autre part, par les références historiques de l'institution, lesquelles influencent les différents publics de l'organisation (Blombäck et Brunninge, 2009).

6 La définition d'une identité de marque solide et cohérente représente un défi, mais aussi une obligation, pour toutes les universités. Ainsi, le processus d'internationalisation qui a lieu dans le milieu académique (programmes d'échanges internationaux pour les élèves et les professeurs, ouverture de filiales de l'université dans plusieurs pays, etc.), la concurrence croissante existante entre les universités autant au niveau de l'ensei gnement que de la recherche, ou encore la baisse de la natalité dans certains pays sont quelques-uns des facteurs qui obligent les universités à fixer leur identité d'une manière claire pour ainsi pouvoir fixer un positionnement performant dans le marché universitaire international. L'identité organisationnelle se compose d'éléments percep tifs, de l'identité souhaitée par l'organisation, de l'identité projetée et de l'identité appliquée (Van Riel et Fombrun, 2007), d'où l'importance que les universités implémentent des mécanismes professionnels leur permettant de gérer leur identité institutionnelle d'une manière performante.

7 Une fois que l'université a défini son identité institutionnelle, elle doit la divulguer auprès de ses stakeholders internes et externes dans le but de créer chez eux une image de marque unique et cohérente. L'image de marque fait référence aux perceptions du consommateur sur la place occupée par une marque dans un marché donné (Herrmann et Huber, 2000), autrement dit, la place occupée par une université dans le marché mondial 
des universités. Par ailleurs, l'image de marque est en rapport avec le comportement de chaque employé de l'université. Tous les employés des organisations, notamment les cadres, doivent considérer l'impact de l'identité, l'image et la réputation et doivent apprendre à gérer ces éléments stratégiques (Argenti, 2003) puisque, finalement, l'image de marque se transmet à travers les actions de communication massive (publicité, relations presse, etc.), mais aussi à travers le comportement de chacun des membres de l'université.

but principal poursuivi par les universités qui parient sur la gestion professionnelle de la communication institutionnelle est la création d'une valeur de marque permettant à l'institution de fixer un positionnement solide dans le marché universitaire. La valeur de marque est un actif intangible qui se base sur le marché dont on peut tirer profit, et qui permet d'améliorer la performance de l'institution (Bick, 2009). Dans le cas des universités, cette valeur peut faire référence à la qualité de l'enseignement, le prestige dans la recherche, la qualité humaine des employés, etc. Néanmoins, le concept de valeur de marque doit être élargi pour ainsi concerner davantage le consommateur (Christopher, 1996). Et, pour ce faire, les universités mettent en place des méthodes de travail exhaustives leur permettant de dénicher quelles sont les valeurs décisives pour leur marque aux yeux du consommateur. Ces méthodes peuvent être internes, comme par exemple la projection de l'identité de marque; et externes, comme par exemple la projection de l'image de marque (De Chernatony et al., 2004).

9 Le travail réalisé par une organisation dans le but de développer sa valeur de marque revêt une valeur stratégique parce que la valeur de marque influence d'une manière positive sur les processus de décision suivis par le consommateur au moment de choisir une marque (Chang et Liu, 2009). D'ailleurs, ces processus de décision sont plus influencés par l'intuition que par la raison (Heath, 2001), et la valeur de marque permet précisément de toucher la capacité intuitive du consommateur.

La promotion de la valeur de marque permet aux universités de développer la »loyauté de marque ». La vraie loyauté de marque est en rapport avec la satisfaction du consommateur, autrement dit, avec l'attitude et le comportement favorables du consommateur envers la marque (Colmenares et Saavedra, 2007). D'ailleurs, la valeur de marque est à la base de la réputation institutionnelle de l'université. La réputation n'est pas une simple impression, mais une vraie évaluation que les groupes d'intérêt font de l'organisation (Cornelissen, 2008). Et la gestion de la réputation représente un terrain d'action stratégique pour les universités puisqu'elles sont en rapport avec plusieurs stakeholders (étudiants, chercheurs, autorités publiques, etc.).

11 Ainsi, la gestion professionnelle de la communication institutionnelle dans une université comporte, en premier lieu, la définition de l'identité de marque ; ensuite, la diffusion de cette identité et la création d'une image de marque chez les stakeholders; et finalement, le développement de cette image de marque dans le but de promouvoir la valeur de marque et ainsi la réputation de l'institution. L'enjeu stratégique que représentent ces éléments identitaires (identité institutionnelle, image de marque, valeur de marque, réputation organisationnelle) justifie la création, au niveau interne de l'université, d'un département de communication institutionnelle qui gère le processus de construction et divulgation de la marque dans le but d'aider l'université à établir un positionnement solide et cohérent dans le marché international des universités. 


\section{Le rôle des réseaux sociaux dans la création de la valeur marque}

12 Lors de ces dernières années, Internet est devenu un outil de communication de plus en plus puissant. Ainsi, $30 \%$ de la population mondiale dispose d'un accès Internet, un chiffre qui s'élève à $70 \%$ dans les pays développés (Internet Telecommunication Union, 2011). Le développement d'Internet s'est accéléré grâce à l'implémentation des réseaux sociaux tels que Facebook, Twitter ou Myspace (Web 2.0). Cette réalité constitue un nouveau défi pour les professionnels de la communication travaillant dans le domaine de la marque institutionnelle. Ainsi, de plus en plus d'annonceurs sont conscients du fait que la structure de la marque résulte d'un engagement raisonnable entre les besoins et les obligations du monde offline et celles du monde online (Strebinger et Treiblmaier, 2004), autrement dit, aujourd'hui, il faut considérer ces deux mondes communicationnels pour bien construire la valeur de marque d'une organisation.

Dans l'actualité, la valeur de marque dépend de plus en plus des consommateurs, lesquels trouvent dans le Web 2.0 un outil qui leur rend plus simple la génération de contenus, la production collective et le partage d'expériences personnelles (Adams, 2010). La participation du consommateur devient fondamentale, notamment parce qu'il est plus satisfait avec l'organisation quand il participe d'une manière active dans la création de la marque (Jamal et Al-Marri, 2007). Dans ce contexte, les universités parient sur une communication participative, personnelle et collaborative. Et pour ce faire, les réseaux sociaux constituent un outil déterminant qui permet aux organisations de promouvoir la participation, la collaboration et l'ouverture (Eysenbach, 2008). Cette approche participative de la communication constitue une innovation dans le milieu de la communication institutionnelle des universités.

En plus de la participation, les réseaux sociaux aident les universités à mettre en place d'autres initiatives leur permettant d'élaborer des stratégies de communication plus créatives et efficaces. Parmi ces initiatives, on peut mettre en avant le nouveau rôle de la personnalisation. Ainsi, l'université cède son pouvoir aux personnes, à savoir, aux employés et aux étudiants, lesquels deviennent les vrais protagonistes de la commu nication institutionnelle universitaire. Le vrai intérêt des réseaux sociaux ne réside pas dans les plateformes techniques, mais plutôt dans les manières créatives qu'ont les usagers de les utiliser (Millerand, 2010), ce qui met encore en valeur l'importance accordée aux personnes. La plupart des usagers d'Internet âgés de moins de 30 ans sont devenus des usagers actifs des réseaux sociaux (Sublet et al., 2011), d'où l'importance que les universités gèrent d'une manière efficace l'arrivée en puissance des personnes au détriment des institutions.

D’un point de vue général, les réseaux sociaux proposent aux universités trois terrains d'action principaux pour promouvoir leur valeur de marque: a) la divulgation d'informations institutionnelles, b) la diffusion d'informations à caractère pédagogique et c) la promotion de certaines actions commerciales.

16 En premier lieu, les réseaux sociaux constituent un support intéressant pour diffuser des informations institutionnelles telles que la mission de l'université, la vision, et les valeurs. La mission aide l'organisation à mieux comprendre les publics ciblés, les priorités dans les actions de communication et le ton du discours communicationnel le plus approprié (Parsons, 2001). La vision représente un niveau de motivation plus élevé que celui de la mission, autrement dit, la mission décrit les moyens, tandis que la vision établit une 
déclaration captivante de ce que l'institution s'efforce d'accomplir (Barret, 2003) ; il s'agit de l'horizon qui guide toutes les actions entreprises par l'université. Par rapport aux valeurs institutionnelles, le but de ces valeurs est de créer dans une entreprise une ambiance basée sur la confiance, la cohésion, l'unité et la communauté (Barret, 2003). La diffusion de ces trois éléments (mission, vision et valeurs) permet à l'université de transmettre une image unique, laquelle doit être cohérente avec son identité pour ainsi pouvoir développer la valeur de marque. La gestion professionnelle et précise de ces trois éléments identitaires évite à l'institution d'avoir plusieurs identités institutionnelles, ce qui pourrait représenter un vrai problème (Van Riel et Fombrun, 2007).

17 En deuxième lieu, la diffusion d'informations à caractère pédagogique, comme par exemple l'organisation de conférences académiques, l'édition de livres ou des revues portant sur les résultats de recherche obtenus par l'université, ou encore la divulgation de vidéos pédagogiques. La divulgation de ce genre d'informations aide l'université à tisser des liens solides avec les différents stakeholders dans le sens où l'université devient un fournisseur de connaissances utiles pour les différentes parties prenantes. Autrement dit, l'université légitime son rôle social. Par ailleurs, ce genre d'actions est en rapport avec la responsabilité sociale de l'université (RSE). La communication de la RSE porte sur plusieurs aspects comme par exemple la mission et les valeurs, les discours, la publicité et la philanthropie ; autrement dit, sur les contributions de l'institution à l'enseignement, la santé, l'assistance et les arts (Cutlip, Center et Broom, 2001). Le recours aux réseaux sociaux pour communiquer la RSE constitue une action appropriée parce que la communication des organisations sur les réseaux sociaux jouit d'un bon niveau de crédibilité (Interactive Advertising Bureau, 2011). Ainsi, par exemple, après la catastrophe subie à Haïti le 12 janvier 2010, la Croix Rouge américaine a reçu plus de cinq millions de dollars grâce aux messages envoyés sur le téléphone portable et sur les réseaux sociaux MySpace et Facebook (Keim et Noji, 2011).

Et finalement, en troisième lieu, la promotion de certaines actions commerciales, autrement dit, la mise en place de mécanismes de marketing dont le but est la promotion des services proposés par l'université (études, recherche, collaborations professionnelles avec les entreprises, etc.). La promotion de ces actions peut contribuer à développer la valeur de marque de l'université, mais pour ce faire il faut considérer préalablement deux idées.

D'une part, le marketing a une dimension externe, mais aussi une dimension interne qui vise la promotion de la créativité et de l'initiative chez l'employé (Almenara Aloy et al., 2005), ce qui doit être présent dans les actions de marketing que les universités mettent en place sur les réseaux sociaux. Et d'une autre part, le marketing est un processus global à travers lequel les institutions créent de la valeur pour leurs clients pour ainsi construire des relations solides avec eux (Kotler et Armstrong, 2008), d'où l'importance que les universités dépassent l'approche du marketing basée sur la vente d'un produit ou service et adoptent une approche de marketing centrée sur la création de valeur. Et pour ce faire, les universités doivent accorder une importance majeure à la communication interpersonnelle sur les réseaux sociaux. La communication interpersonnelle influence sur la génération de motivation chez les personnes qui participent dans le processus communicationnel (Duterme, 2007), met en valeur l'importance de savoir écouter (Ongallo, 2007) et permet d'améliorer l'efficacité de la communication grâce à la présentation individuelle de l'information et au retour immédiat (Kotler, Shalowitz et Stevens, 2008). 


\section{L'impact de Facebook sur la valeur de marque} niveau international. On estime que le nombre d'usagers de Facebook en mars 2012 était de 835525280 personnes, dont 232835740 en Europe, 195034380 en Asie, 173284940 en Amérique du Nord et 112531100 en Amérique du Sud (Internet World Stats, 2013). Il s'agit d'un nouveau média très intéressant pour les entreprises, y compris les universités, notamment si on considère quelques-unes des statistiques le concernant. Ainsi, d'une part, $80 \%$ des usagers des réseaux sociaux préfèrent entrer en contact avec les marques à travers Facebook; et d'une autre part, $42 \%$ des professionnels du marketing considèrent que Facebook est un outil très important pour les affaires de l'entreprise ainsi que pour la mise en place de campagnes de marketing (Business2Community, 2013), et tout spécialement pour la mise en place d'actions de marketing viral (Gjoka et al., 2009). Les réseaux sociaux, comme Facebook, persuadent chaque jour des millions de personnes pour adopter certains comportements (Fogg et Iizawa, 2008), c'est pourquoi de plus en plus d'entreprises utilisent ce support pour le développement de leur stratégie de relations publiques (Waters et al., 2009). Autrement dit, Facebook est d'ores et déjà un média déterminant pour les campagnes de marketing, communication et relations publiques des entreprises, et ceci dans n'importe quel pays du monde.

gré l'intérêt de Facebook comme outil de marketing et de communication, il ne faut pas oublier que les nouvelles technologies ne sont importantes que quand elles arrivent à changer la manière d'agir des personnes, et surtout, les manières qu'ont ces personnes d'agir ensemble (Grimmelmann, 2009). D'où l'importance de comprendre quel est le comportement des usagers sur Facebook.

Pour les usagers, Facebook constitue un support très intéressant pour promouvoir l'interaction et la communication entre les personnes, mais il représente aussi un problème au niveau de la sécurité des données diffusées (Acquisti et al., 2006). D’un point de vue général, on peut affirmer que les usagers de Facebook sont plus confiants que les usagers de Myspace en ce qui concerne la diffusion d'informations personnelles (Dwyer et al., 2007). Par ailleurs, les usagers de Facebook sont des personnes un peu extraverties et narcissiques, mais aussi ce sont des gens qui ne sont pas solitaires (Ryan et Xenos, 2011). En ce qui concerne leur comportement, il faut dire que, d'une part, les identités construites sur Facebook diffèrent de celles que les usagers construisent sur d'autres sites internet où ils peuvent garder l'anonymat (Zhao et Grasmuck, 2008); et d'une autre part, l'expérience préalable avec la page de Facebook ainsi que les différences culturelles ont un effet sur les motivations des usagers de Facebook ainsi que sur l'usage réalisé du réseau social et le temps investi (Vasalou et al., 2010). Finalement, il faut mettre en avant le fait que la plupart des usagers des réseaux sociaux associent Facebook avec l'amusement et avec le fait d'être au courant des activités sociales qui ont lieu dans l'entourage de ce réseau social (Quan-Haase et Young, 2010).

Facebook constitue un nouveau média global qui permet la diffusion d'actions de marketing, communication et relations publiques. Mais ce média utilise un nouveau langage caractérisé par le protagonisme du consommateur, le rôle stratégique de l'interaction et l'importance accordée à l'amusement. Ces aspects doivent être considérés par les universités qui parient sur Facebook comme un outil leur permettant d'enrichir leur valeur de marque. La possibilité d'établir une communication interpersonnelle et

Quaderni, 85 | Automne 2014 
bidirectionnelle entre l'université et ses différents stakeholders, la richesse audiovisuelle de ce média (diffusion d'images, vidéos, textes, voix, etc.) ainsi que la dimension internationale justifient la décision de plusieurs universités d'avoir recours à Facebook pour construire et promouvoir leur valeur de marque.

Méthodologie

L'objectif de cet article est de savoir si les réseaux sociaux contribuent à la création de la valeur de marque des universités. Et plus précisément, on cherche à savoir si Facebook, le réseau social le plus réputé au niveau mondial, aide à la construction de marque des institutions universitaires. Pour ce faire, on a réalisé une analyse quantitative basée sur l'étude du classement mondial des universités Academic Ranking of World Universities, élaboré par le Center for World-Class Universities et l'Institute of Higher Education of Shanghai (Jiao Tong University, Chine). En 2012, ces organismes ont analysé 500 universités partout dans le monde, et en plus ont élaboré des classements spécialisés selon le domaine des connaissances, comme par exemple les meilleures universités dans le domaine de l'économie, les mathématiques ou la médecine. Parmi tous les classements proposés par ces organismes, on a choisi le classement des 500 meilleures universités au niveau mondial, et on a centré l'analyse sur les 100 meilleures universités ${ }^{1}$. On a choisi uniquement les cent meilleures universités parce que dans ce classement, à partir du poste numéro 101 les universités qui obtiennent des ponctuations similaires sont groupées en différents blocs de cinquante universités et elles sont classées par ordre alphabétique à l'intérieur de ces blocs.

L'analyse réalisée se base sur l'étude du profil Facebook des cent meilleures universités dans le monde. L'analyse de chaque page web s'est faite en considérant dix critères différents permettant d'évaluer si le profil Facebook aide l'université à construire et à divulguer sa valeur de marque. Le choix des dix critères s'est basé sur les trois terrains d'action que les réseaux sociaux proposent aux universités pour promouvoir leur valeur de marque : a) la divulgation d'informations insti tutionnelles, b) la promotion de certaines actions commerciales, et c) la diffusion d'informations à caractère pédagogique. Les dix critères sont groupés dans trois blocs qui représentent ces trois terrains d'action (voir ces trois blocs dans la rubrique Résultats de l'étude).

\section{Résultats de l'étude}

Pour faire cette analyse, on est parti de deux principes: l'ordre et l'approche internationale. C'est pourquoi, on a considéré uniquement les informations bien organisées dans chaque rubrique et non pas celles qui sont éparpillées d'une manière désorganisée sur le » mur» de Facebook. Ainsi, par exemple, on a analysé les vidéos groupées dans la rubrique " vidéo " ou » Youtube ", mais non pas les vidéos qui sont divulguées dans la rubrique » mur ». Par ailleurs, puisque la plupart des cent meilleures universités sont présentes dans des pays anglophones et que l'approche internationale pour créer la valeur de marque exige l'usage de l'anglais, on a considéré uniquement la version en anglais du profil Facebook de chaque université. Sur les cent meilleures universités du monde, il y en a 85 qui disposent d'un profil sur Facebook, institutionnel et 
en anglais. Dans le reste d'universités, soit le profil n'est pas actif (il n'y a pas de contenu, ou la page n'existe même pas), soit les universités disposent de » groupes » ou de pages individuelle pour une faculté, mais non pas pour l'ensemble de l'université. C'est pourquoi, on a centré l'analyse sur 85 universités.

Les dix critères utilisés pour analyser le profil Facebook des cent meilleures universités du monde peuvent se grouper dans trois blocs différents : a) la divulgation d'informations institutionnelles, b) la promotion de certaines actions commerciales, et c) la diffusion d'informations à caractère pédagogique.

En premier lieu, la divulgation d'informations institutionnelles. Afin d'évaluer ce bloc, on s'est basé sur l'information disponible sur la rubrique de Facebook » À propos » et on a utilisé trois critères : a) existence d'une description de l'organisation, b) description de la mission de l'organisation et c) existence de liens vers d'autres pages web institutionnelles de l'université (page officielle, Youtube, etc.). Selon cette étude, 88,20 \% des hôpitaux décrivent l'organisation; $28,20 \%$ décrivent la mission de l'institution; et 94,10\% disposent de liens vers d'autres pages institutionnelles de l'université, comme par exemple la page officielle de l'organisation ou le profil sur Youtube.

En deuxième lieu, la promotion de certaines actions commerciales. Pour ce faire, on a analysé la rubrique Facebook » Mur » et on a considéré trois critères : a) présence du logo à côté de l'image principale du profil, b) existence, juste au-dessous de l'image principale du profil, d'un texte incitant les étudiants à s'inscrire à l'université et c) organisation des groupes de discussion selon un sujet précis (innovation, évènements qui on lieu à l'université, etc.). Selon les chiffres apportés par cette analyse, $83,50 \%$ des universités placent leur logo juste à côté de l'image principale du profil ; 9,40\% des universités ajoutent, juste au-dessous de l'image principale du profil, un texte incitant les étudiants à s'inscrire à l'université ; et $87,10 \%$ des institutions organisent les groupes de discussion selon des sujets précis, tels que, par exemple, les évènements qui ont lieu à l'université (conférences, cours, évènements sportifs, etc.).

28 Et en troisième lieu, la diffusion d'informations à caractère pédagogique. Pour analyser ce sujet, on a considéré plusieurs rubriques de Facebook et plusieurs critères. Ainsi, sur la rubrique » Vidéos »,on a considéré deux critères : a) existence de vidéos pour divulguer des connaissances académiques ou scientifiques (par exemple, entretiens à des professeurs); et b) présence de commentaires juste au-dessous de chaque vidéo. D’ailleurs, sur la rubrique " Articles ", on a considéré ce critère : a) existence de liens vers des articles à caractère académique, autrement dit, des textes scientifiques. Et, finalement, sur la rubrique » Évènements », on a suivi ce critère :

a) diffusion d'informations sur des évènements académiques qui auront lieu à l'université, comme par exemple des cours ou des conférences. Selon cette étude, 51,80\% des universités disposent de vidéos dont le but est la diffusion des connaissances académiques; $40 \%$ des universités disposent de vidéos académiques sur lesquels les visiteurs réalisent des commentaires ; 10,60\% des universités proposent des liens vers des articles scientifiques; et $32,90 \%$ des organisations renseignent les visiteurs du profil Facebook sur les évènements académiques qui auront lieu à l'université, comme par exemple les conférences, les cours ou les soutenance de thèse. D'ailleurs, on peut signaler que $67,06 \%$ des universités respectent entre 5 et 8 critères (voir Tableau 2. Pourcentage de critères respectés), que Brown University est l'université qui respecte le plus de critères (voir Tableau 2. Universités respectant au moins $80 \%$ des critères) et que les trois critères les 
plus respectés par les universités sont, dans cet ordre : a) existence de liens vers d'autres pages institutionnelles de l'université, b) description de l'organisation et c) existence de groupes de discussion bien organisés selon un sujet précis (voir Tableau 3. Critères les plus respectés).

\section{Discussion}

L'objectif de cet article est d'étudier l'influence de Facebook sur la construction de la valeur de marque des universités. Malgré l'intérêt de certaines informations apportées par cette étude, il y a quelques limitations qui peuvent être signalées comme par exemple le manque d'informations sur les perceptions des stakeholders de l'université (étudiants, professeurs, etc.) sur le profil Facebook et sur la marque de l'université, l'impossibilité de connaître la stratégie de communication institutionnelle utilisée par chaque université, ou encore le manque d'informations sur la stratégie suivie par chaque université pour intégrer la communication réalisée sur Facebook avec celle réalisée sur les autres applications online, telles que, par exemple, la page Web de l'université ou le profil de l'organisation sur d'autres réseaux sociaux comme MySpace ou Twitter. Étant donné le contexte actuel des universités et le pari ferme que ces organisations ont réalisé sur le développement de la communication online 2.0, il s'avère intéressant que d'autres chercheurs continuent de développer ce milieu, notamment les aspects clés comme par exemple l'intégration de Facebook avec les applications de la téléphonie mobile, les rapports existant entre l'implication des étudiants avec Facebook et le sentiment d'appartenance à l'université, ou encore le rôle de Facebook comme outil de diffusion de connaissances académiques.

Si on analyse les résultats obtenus, on peut affirmer que les universités consacrent une im portance croissante à la communication online à travers Facebook, laquelle peut contribuer à créer et développer la valeur de marque de l'institution. Ainsi, le fait que $83,50 \%$ des universités place le logo de l'institution à côté de l'image principale du profil Facebook, ou encore que 94,10\% des universités analysées dispose de liens vers d'autres pages où l'université a une présence institutionnelle (Youtube, MySpace, Twitter, etc.) met en évidence le pari ferme des organisations universitaires pour la création de la valeur de marque à travers les applications 2.0. L'interaction proposée par les réseaux sociaux ainsi que la possibilité de partager contribue à renforcer la vitalité d'une marque, et donc, à créer la valeur de marque (Jensen et al., 2009), d'où le fait que les universités accordent plus d'importance aux outils capables de générer cet interaction, comme par exemple les commentaires réalisés sur les vidéos ( $40 \%$ des universités dispose de ces com mentaires) ou la diffusion d'informations sur les évènements à caractère pédagogique (32,90\% des universités diffuse ce genre d'informations).

Par ailleurs, il faut souligner le fait que la communication des attributs de marque à travers la mission et la vision constitue une action stratégique pour créer la valeur de marque (Ingenhoff et Fuhrer, 2010), c'est pourquoi c'est étonnant que seulement 28,20\% des universités décrive d'une manière claire et précise la mission de l'organisation. Finalement, il faut mettre en avant le manque d'actions de communication centrées sur l'aspect académique. Ainsi, 51,80\% des universités divulgue des vidéos portant sur des sujets académiques, 32,90 \% des universités renseigne sur les évènements académiques et seulement $10,60 \%$ des universités diffuse des articles académiques. Cette réalité constitue un vide communicationnel préoccupant dans le sens où le point fort des universités est la 
création de connaissances acadé.miques ou scientifiques, lesquelles doivent être divulguées et partagées avec les différentes parties prenantes (étudiants, professeurs, journalistes, etc.)

\section{Conclusion}

En introduction, on s'est posé cette question : quel est l'impact de la communication 2.0 sur la construction de marque des universités? Après avoir réalisé une révision bibliographique sur le sujet ainsi qu'une analyse quantitative sur le profil Facebook des cent meilleures universités au monde, on peut dire que la communication réalisée à travers Facebook contribue au développement de la valeur de marque des universités en raison de deux réalités différentes, mais complémentaires. En premier lieu, la construction de la valeur de marque se base sur l'identité de l'organisation, mais aussi sur les perceptions qu'ont les différentes parties prenantes sur cette organisation, d'où l'importance de disposer d'un outil, comme Facebook, capable d'apporter à l'université des informations lui permettant de comprendre quel est l'imaginaire que chaque stakeholder a construit sur l'image de l'université. Et, en deuxième lieu, Facebook constitue un outil de communication qui permet à l'individu de devenir le protagoniste de l'institution, apporter des idées, partager des informations et des expériences; autrement dit, Facebook rend possible la construction collective de la marque entre l'institution et les parties prenantes, ce qui contribue à créer une marque solide et crédible.

Tableau 1. Pourcentage de critères respectés

\begin{tabular}{|c|c|c|}
\hline Nombre de critères respectés & Nombre d'universités & Pourcentage d'universités \\
\hline 10 & 0 & $0 \%$ \\
\hline 9 & 1 & $1,18 \%$ \\
\hline 8 & 9 & $10,59 \%$ \\
\hline 7 & 15 & $17,65 \%$ \\
\hline 6 & 14 & $16,47 \%$ \\
\hline 5 & 19 & $22,35 \%$ \\
\hline 4 & 16 & $18,82 \%$ \\
\hline 3 & 3 & $3,53 \%$ \\
\hline 2 & 4 & $4,71 \%$ \\
\hline 1 & 1 & $1,18 \%$ \\
\hline 0 & 3 & $3,53 \%$ \\
\hline Total & 85 & $100 \%$ \\
\hline
\end{tabular}


Tableau 2. Universités respectant au moins le $80 \%$ des critères

\begin{tabular}{|l|l|}
\hline Université & Nombre de critères \\
\hline Brown University & 9 \\
\hline Stanford University & 8 \\
\hline Massachusetts Institute of Technology & 8 \\
\hline Columbia University & 8 \\
\hline University of Colorado at Boulder & 8 \\
\hline Pennsylvania State University - University Park & 8 \\
\hline Carnegie Mellon University & 8 \\
\hline King's College London & 8 \\
\hline Technion - Israel Institute of Technology & 8 \\
\hline University of Nottingham & 8 \\
\hline
\end{tabular}

Tableau 3. Critères les plus respectés

\begin{tabular}{|l|l|}
\hline Critère & $\begin{array}{l}\text { Pourcentage d'universités respectant ce } \\
\text { critère }\end{array}$ \\
\hline $\begin{array}{l}\text { 1. Liens vers d'autres pages institutionnelles } \\
\text { (Youtube, etc.) }\end{array}$ & $94,10 \%$ \\
\hline 2. Description de l'organisation & $88,20 \%$ \\
\hline 3. Groupes de discussion selon sujet & $87,10 \%$ \\
\hline $\begin{array}{l}\text { 4. Présence du logo à côté de l'image principale du } \\
\text { profil }\end{array}$ & $83,50 \%$ \\
\hline 5. Vidéos pour divulguer des connaissances & $51,80 \%$ \\
\hline 6. Vidéos commentées par les visiteurs & $40 \%$ \\
\hline 7. Informations sur des évènements à caractère \\
pédagogique
\end{tabular}


10. Texte incitant les étudiants à s'inscrire à

l'université

$9,40 \%$

\section{BIBLIOGRAPHIE}

ACQUISTI, Alessandro, GROSS, Ralph. « Ima-gined communities : awareness, information sharing, and privacy on the facebook ", in Golle, P. et Danezis, G. (dir.), Proceedings of 6th Workshop on Privacy Enhancing Technologies. Cambridge : Robinson College, 2006, pp. 36-58.

ADAMS, Samantha. » Revisiting the online health information reliability debate in the wake of web 2.0 : an interdisciplinary literature and website review ». International Journal of Medical Informatics, $\mathrm{n}^{\circ}$ 79, v. 6, 2010, pp. 391-400.

ÄHLSTRÖM, Jenny. « Corporate response to CSO criticism : decoupling the corporate respon sibility discourse from business practice ». Corporate Social Responsibility and Environmental Management, no 17, v. 2, 2010, pp. 70-80.

ALMENARA ALOY, Jaume, ROMEO DELGADO, Marina, ROCA PÉREZ, Xavier (2005). Comunicación interna en la empresa. Barcelona : Editorial UOC.

ARGENTI, Paul. Corporate communication. Boston : McGraw-Hill/Irwin, 2003.

BALMER, John. « Identity based views of the corporation. Insights from corporate identity, organisational identity, social identity, visual identity, corporate brand identity and corporate image ». European Journal of Marketing, no 42, v. 9/10p 2008, pp. 879-906.

BARRETT, Richard. Libérer l'âme de l'entreprise. Bâtir une organisation visionnaire guidée par les valeurs . Bruxelles : De Boeck, 2003.

BICK, Geoffrey. « Increasing shareholder value through building customer and brand equity ». Journal of Marketing Management, nº 25, v. 1-2, 2009, pp. 117-141.

BLOMBÄCK, Anna, BRUNNINGE, Olof. « Corporate identity manifested through historical references. Corporate Communications : An International Journal, nº 14, v. 4, 2009, pp. 404-419.

BUSINESS2COMMUNITY. «Facebook marketing statistics you need to know », [en ligne], http:// www.business2community.com/facebook/facebook-marketing-statistics-you-need-toknow-0289953, page consultée le 12 mars 2013.

CHANG, Hsin, LIU, Ya. « The impact of brand equity on brand preference and purchase intentions in the service industries ». The Service Industries Journal, no 29, v. 12, 2009, pp. 1687-1706.

CHRISTOPHER, Martin. « From brand values to customer value ». Journal of Marketing Practice : Applied Marketing Science, nํㅡㄴ, v. 1, 1996, pp. 55-66.

COLMENARES, Oscar, SAAVEDRA, José. « Aproximación teórica de la lealtad de marca : enfoques y valoraciones ». Cuadernos de Gestión, nำ 7, v. 2, 2007, pp. 69-81.

CORNELISSEN, Joep. Corporate communication : a guide to theory and practice. Los Angeles : Sage, 2008. 
CUTLIP, Scott, CENTER, Allen, BROOM, Glen. Relaciones públicas eficaces (Trad. E. Smyrli). Barcelona : Gestión 2000.

DE CHERNATONY, Leslie, DURY, Susan, SEGAL-HORN, « Susan. Identifying and sustaining services brand's values ». Journal of Marke-ting Communication, no 10, v. 2, 2004, pp. 73-93.

DUTERME, Claude. La communication interne en entreprise : l'approche de Palo Alto et l'analyse des organisations. Bruxelles : De Boeck Université, 2007.

DWYER, Catherine, HILTZ, Starr et PASSERINI,Katia. « Trust and privacy concern within social networking sites : a comparison of Facebook and MySpace ». Proceedings of the Thirteenth Americas conference on information systems. Colorado : AMCIS, 2007, pp. 1-12.

EYSENBACH, Gunther. « Medicine 2.0 : social networking, collaboration, participation, apo mediation, and openness ». Journal of Medical Internet Research, no 10, v. 3, 2008, p. e22.

FOGG, B., IIZAWA, Daisuke. « Online persuasion in Facebook and Mixi : a cross-cultural comparison », in Oinas-Kukkonen et al. (dir.), Persuasive Technology. New York : Springer, 2008, pp. 35-46.

GJOKA, Minas, KURANT, Maciej, BUTTS, Carter, MARKOPOULOU, Athina. « A walk in Facebook : uniform sampling of users in online social networks ». The Computing Research Repository, no 906, 2009.

GRIMMELMANN, James. Saving Facebook. Iowa Law Review, no 94, 2009, pp. 1137-1206.

HARRIS, Fiona, DE CHERNATONY, Leslie. « Corporate branding and corporate brand per formance ». European Journal of Marketing, nº 35, v. 3-4, 2001, pp. 441-456.

HEATH, Robert. « Low involvement processing - a new model of brand communication ». Journal of Marketing Communications, $\mathrm{n}^{\circ}$ 7, v. 1, 2001, pp. 27-33.

HERRMANN, Andreas, HUBER, Frank. « Value-oriented brand positioning ». The International Review of Retail, Distribution and Consumer Research, no10, v. 1, 2000, pp. 95-112.

INGENHOFF, Diana, FUHRER, Tanja. « Posi-tioning and differentiation by using brand personality attributes : Do mission and vision statements contribute to building a unique corporate identity? ». Corporate Communications : An International Journal, nº 15, v. 1, 2010, pp. 83-101.

INTERACTIVE ADVERTISING BUREAU. El libro Blanco IAB. La comunicación en medios sociales. Madrid : Interactive Advertising Bureau, 2011.

INTERNET TELECOMMUNICATION UNION. The World in 2011 - ICT facts and figures. Geneva :Internet Telecommunication Union, 2011.

INTERNET WORLD STATS. « Facebook users in the world », [en ligne], http:// www.internetworldstats.com/facebook.htm, page consultée le 25 mars 2013.

JAMAL, Ahmad, AL-MARRI, Mohammed. « Exploring the effect of self-image congruence and brand preference on satisfaction : the role of expertise ». Journal of Marketing Management, 는 23, v. 7-8, 2007, pp. 613-629.

JANISZEWSKI, Chris, VAN OSSELAER, Stijn. « A connectionist model of brand-quality associa tions ». Journal of Marketing Research, nº 37, v. 3, 2000, pp. 331-350.

JENSEN, Hope, MUÑIZ, Albert, ARNOULD, Eric. « How brand community practices create value ». Journal of Marketing, no 73, v. 5, 2009, pp. 30-51.

KEIM, Marc, NOJI, Eric. « Emergent use of social media : a new age of opportunity for disaster resilience ». American Journal of Disaster Medicine, no 6, v. 1, 2011, pp. 47-54. 
KOTLER, Patrick, ARMSTRONG, Gary. Principles of marketing (12st Ed.). Upper Saddle River, New Jersey : Pearson/Prentice Hall, 2008.

KOTLER, Philip, SHALOWITZ, Joel, STEVENS, Robert. Strategic marketing for health care organizations : building a customer-driven health system. San Francisco : Jossey-Bass, 2008.

MILLERAND, Florence. Web social : mutation de la communication. Montréal : Presses de l'Université du Québec, 2010.

ONGALLO, Carlos. Manual de comunicación : guía para gestionar el conocimiento y las relaciones humanas en empresas y organizaciones. Madrid : Dyckinson, 2007.

PARSONS, Patricia. Beyond persuasion : the healthcare manager's guide to strategic communication. Chicago : Health Administration Press, 2001.

QUAN-HAASE, Anabel, YOUNG, Alyson. « Uses and gratifications of social media : a comparison of Facebook and instant messaging ». Bulletin of Science Technology Society, ํㅜ 30, v. 5, 2010, pp. 350-361.

RYAN, Tracii, XENOS, Sophia. « Who use Facebook. An investigation into the relationship between the big five, shyness, narcissim, loneliness, and Facebook usage ». Computer in Human Behaviour, no 27, v. 5, 2011, pp. 1658-1664.

STREBINGER, Andreas, TREIBLMAIER, Horst. «E-Adequate branding : building offline and online brand structure within a polygon of interdependent forces ». Electronic Markets, ํㅡㄹ 14, v. 2, 2004, pp. 153-164.

SUBLET, V., SPRING, C., HOWARD, J. « Does social media improve communication ? Evaluating the NIOSH science blog ». American Journal of Industrial Medicine, no 54, v. 5, 2011, pp. 384-394.

VAN RIEL, Cees, FOMBRUN, Charles. Essentials of corporate communication : implementing practices for effective reputation gestion. Abingdon, Oxon : Routledge, 2007.

VASALOU, A., JOINSON, A., COURVOISIER, D. « Cultural differences, experience with social networks and the nature of "true commitment" in Facebook ». International Journal of HumanComputer Studies, no 68, 2010, pp. 719-728.

WATERS, R., BURNETT, E., LAMM, A., LUCAS, J. « Engaging stakeholders through social networking : How nonprofit organizations are using Facebook ». Public Relations Review, nº 35, v. 2, 2009, pp. 102-106.

WILSON, Alan. « Understanding organisational culture and the implications for corporate mar keting ». European Journal of Marketing, nº 35, v. 3-4, 2011, pp. 353-367.

ZHAO, Shanyang, GRASMUCK, Martin. «Identity Construction on Facebook :

DigitalEmpowerment in Anchored Relationships ». Computers in Human Behavior, nํ2 24, v. 5, 2008, pp. 1816-1836.

\section{NOTES}

1. Selon le classement 2012, les cent meilleures universités au niveau mondial étaient dans cet ordre: 1) Harvard University; 2) Stanford University; 3) Massachusetts Institute of Technology (MIT); 4) University of California, Berkeley; 5) University of Cambridge; 6) California Institute of Technology; 7) Princeton University; 8) Columbia University; 9) University of Chicago; 10) University of Oxford; 11) Yale University; 12) University of California, Los Angeles; 13) Cornell University; 14) University of Pennsylvania; 15) University of California, San Diego; 16) University 
of Washington; 17) The Johns Hopkins University; 18) University of California, San Francisco; 19) University of Wisconsin - Madison; 20) The University of Tokyo; 21) University College London; 22) University of Michigan - Ann Arbor; 23) Swiss Federal Institute of Technology Zurich; 24) The Imperial College of Science, Technology and Medicine; 25) University of Illinois at UrbanaChampaign; 26) Kyoto University; 27) New York University; 28) University of Toronto; 29) University of Minnesota, Twin Cities; 30) Northwestern University; 31) Washington University in St. Louis; 32) Rockefeller University; 33) University of Colorado at Boulder; 34) University of California, Santa Barbara; 35) The University of Texas at Austin; 36) Duke University; 37) University of Paris Sud (Paris 11); 38) University of Maryland, College Park; 39) University of British Columbia; 40) The University of Manchester; 41) University of North Carolina at Chapel Hill; 42) Karolinska Institute; 43) Pierre and Marie Curie University - Paris 6; 44) University of Copenhagen; 45) University of California, Irvine; 46) University of Southern California; 47) University of California, Davis; 48) The University of Texas Southwestern Medical Center at Dallas; 49) Pennsylvania State University - University Park; 50) Vanderbilt University; 51) Carnegie Mellon University; 52) The University of Edinburgh; 53) Technical University Munich; 54) The Hebrew University of Jerusalem; 55) Utrecht University; 56) Purdue University - West Lafayette; 57) University of Melbourne; 58) University of Pittsburgh; 59) University of Zurich; 60) University of Munich; 61) Rutgers, The State University of New Jersey - New Brunswick; 62) University of Heidelberg: 63) McGill University; 64) The Australian National University; 65) Brown University; 66) The Ohio State University - Columbus; 67) University of Oslo; 68) King's College London; 69) University of Geneva; 70) University of Bristol; 71) Boston University; 72) University of Florida; 73) Ecole Normale Superieure - Paris; 74) Leiden University; 75) University of Helsinki; 76) Uppsala University; 77) University of Arizona; 78) Technion-Israel Institute of Technology; 79) Arizona State University - Tempe; 80) Moscow State University; 81) Stockholm University; 82) University of Utah; 83) Osaka University; 84) Indiana University Bloomington; 85) University of Basel; 86) Aarhus University; 87) University of Nottingham; 88) University of Rochester; 89) Ghent University; 90) The University of Queensland; 91) Rice University; 92) McMaster University; 93) Texas A\&M University - College Station; 94) University of Sydney; 95) Weizmann Institute of Science; 96) Michigan State University; 97) Nagoya University; 98) The University of Western Australia; 99) Case Western Reserve University; et 100) University of Freiburg. L'analyse des pages Web a été réalisée du 5 avril 2013 au 29 mai 2013.

\section{RÉSUMÉS}

L'objectif de cet article est de savoir si les réseaux sociaux contribuent à développer la valeur de marque des universités. Pour ce faire, on a réalisé une analyse bibliographique sur le rôle des réseaux sociaux dans la construction de marque ; ainsi qu'une analyse quantitative sur le profil Facebook des cent meilleures universités au monde à l'aide de dix indicateurs. La conclusion de l'article est que Facebook aide les universités à développer leur valeur de marque grâce à sa capacité pour réaliser une construction collective de la valeur de marque entre l'institution et ses stakeholders.

This paper aims to analyze if social media help universities to develop their brand value. To do this, we conducted a literature review on the role of social media in the construction of brand value, and a quantitative analysis on the Facebook profile of the hundred best universities in the 
world using ten indicators. The conclusion of this paper is that Facebook helps universities to develop their brand value through its ability to achieve a collective construction of brand value between the institution and its stakeholders.

\section{AUTEUR}

\section{PABLO MEDINA AGUERREBERE}

Faculté de Communication, Université Internationale de Catalogne 